\title{
CULTURA DIGITAL EN EL BENEMÉRITO CUERPO DE BOMBEROS DE LA REPÚBLICA DE PANAMÁ
}

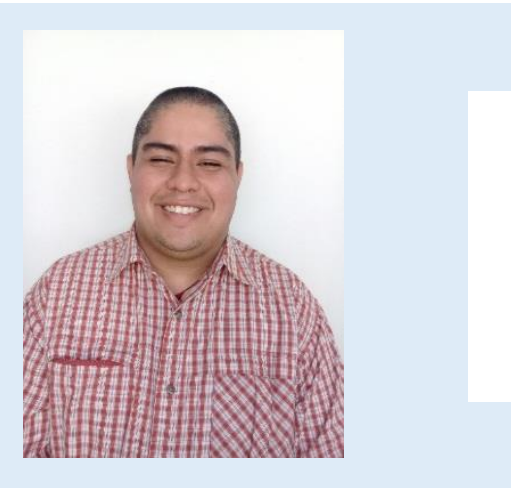

Fecha de recepción: 20/05/2017 Fecha de revisión: 21/07/2017

\section{JOSÉ ROGELIO FUNG CORRO}

Universidad Metropolitana de Educación, Ciencia y Tecnología, Panamá.

investigación.santiago@umecit.edu.pa

\section{RESUMEN}

Uno de los más importantes y significativos temas que acontecen en el entorno y contexto tecnológico y socio-cultural de la nueva era en la cual ha proyectado los más grandes logros científicos de la humanidad, fue la migración de tecnología análoga a tecnología digital. En una perspectiva que ha generado enormes intentos por la búsqueda que evidencian y reconocer el entorno cotidiano que vive una de las Instituciones más prestigiosa de nuestro país, en materia de salvaguardar vidas, bienes y propiedades, como parte de su misión y visión institucional que despierta un enorme interés científico desde como lo son: la química del fuego así como y el uso de las herramientas tecnológicas para fortalecer el desarrollo humano y mejorar las condiciones laborales de las diferentes secciones del Benemérito Cuerpo de Bomberos de la República de Panamá. La Cultura Digital es de suma importancia para la sensibilización y concientización de los usuarios como principales protagonistas para contrarrestar la tecnofobia y erradicar el analfabetismo tecnológico como principales problemas que aquejan esta era digital en nuestra sociedad.

Palabras clave: Cultura Digital, Benemérito Cuerpo de Bomberos de la República de Panamá, analfabetismo tecnológico, era digital. 


\title{
DIGITAL CULTURE IN THE FIRE DEPARTMENT REPUBLIC OF PANAMA
}

\begin{abstract}
One of the most important and significant themes that occur in the technological and sociocultural environment and context of the new era in which it has projected the greatest scientific achievements of humanity was the migration of analog technology to digital technology in a perspective that has generated enormous attempts to find evidence and recognize the daily environment that lives one of the most prestigious institutions of our country, in terms of safeguarding lives, property and property, as part of its mission and institutional vision that awakens a huge scientific interest as they are: the chemistry of fire as well as the use of technological tools to strengthen human development and improve the working conditions of the different sections of the Fire Department of the Republic of Panama. The Digital Culture is of utmost importance for the sensitization and awareness of the users as main protagonists to counter technophobia and to eradicate the technological illiteracy as the main problems that this digital age is in our society. The first attempts to crystallize the idea of a bomberil organization go back to 1870 , when the first voluntary firefighting brigade was organized, determined to confront the constant conflagrations of the time.
\end{abstract}

Keywords: Digital Culture, Fire Department of the Republic of Panama. Technological illiteracy, digital age.

\section{INTRODUCCIÓN}

En la nueva era de la información y el conocimiento, se exigen nuevas competencias entre ellas el uso de herramientas y manejo de contenidos digitales, en estos tiempos de cambio se hacen referencias notables, cuyas repercusiones que han traído las nuevas tecnologías, entre aportes y exigencias a los mercados en tiempos actuales.

En el Benemérito Cuerpo de Bomberos de la República de Panamá, se han vivido muchos cambios y transformaciones, que datan desde antes de que se formara la República de Panamá, en el año 1887.

La Cultura Digital es un tema esencial para mejorar los diversos puntos que fortalecen nuestra Institución, en este contexto donde las tecnologías digitales, el ciberespacio, la hipertextualidad e hipermeabilidad, la inmediatez, la exclusión y la hiperconectividad, son 
algunos temas de prioridad para esta nueva generación de usuarios que vive el sector público y privado en Panamá.

El Benemérito Cuerpo de Bomberos de la República de Panamá, mediante la Dirección Nacional de Tecnología, ha implementado nuevas herramientas de tecnologías de información y comunicación, para incrementar la productividad y fortalecer el rendimiento de las labores del personal para salvaguardar vidas y bienes, en la sociedad panameña, sobre todo en la respuesta a las emergencias que se atienden a diario, que ponen en riesgo la vida de los panameños.

El presente estudio está estructurado en bases explicativas y descriptivas, las cuales fundamentan un completo análisis en cada una de las estaciones locales y Zonas Regionales del Benemérito Cuerpo de Bomberos de la República de Panamá, a nivel tecnológico y se han llevado a cabo con el fin de mejorar la productividad en todos los ámbitos que corresponden al aspecto socio-cultural, con un tema que debe marcar el primer paso al estudio y la sensibilización de la Cultura Digital.

La importancia institucional de desarrollo humano, es crear una cultura digital y a su vez generar conocimiento, como parte de un profundo proceso que cuyo propósito es transformar las ideas en acciones. A su vez, trazar nuevos lineamientos para crear agentes de cambio y de cultura digital organizacional, como parte de un proceso que eleve el Benemérito Cuerpo de Bomberos de la República de Panamá, como una de las instituciones que asuma nuevos retos y desafíos en materia científica, bomberil y académica. Para cumplir con las normas y nuevos modelos que son de suma importancia para el acontecer que se vive en la Institución, para poner en marcha una cultura digital que ha de crear y asumir nuevas responsabilidades organizacionales importantes para construir y transformar los principales ejes de cambio tecnológico del Benemérito Cuerpo de Bomberos de la República de Panamá.

Dentro del compromiso y desarrollo científico, la Dirección Nacional de Tecnología de la institución, ha realizado grandes aportes en la Implementación de Herramientas Tecnológicas que permiten sensibilizar y concientizar a los bomberos sobre la Importancia de la Cultura Digital, en las diferentes Estaciones, donde la Infraestructura Tecnológica, Aplicaciones, Equipos y las nuevas aulas y plataformas virtuales e-Liaoning para la Formación, son esencial para los Bomberos en pleno siglo XXI, donde existe una revolución digital en nuestros días . En esta investigación se pretende lograr un estudio significativo sobre la Cultura Digital dentro 
del Benemérito Cuerpo de Bomberos de la República de Panamá, tomando en cuenta, el impacto y las influencias de las nuevas tendencias de las herramientas utilizadas y a su vez por implementar en los próximos años, sus repercusiones e implicaciones en la sociedad y por ende en la Institución, donde cumplir con la misión y visión de la Institución, sean parte de las herramientas a emplear que satisfacen las necesidades de los Bomberos y su legado Institucional, en el país. (Plan Digital 2015).

La naturaleza de este estudio investigativo es revisar los componentes tecnológicos referidos al ecosistema del Benemérito Cuerpo de Bomberos de la República de Panamá, a nivel nacional y las 11 Zonas Regionales a nivel provincial entre las cuales se tienen: Zona Regional de Panamá, Zona Regional de Panamá Oeste, Zona Regional de Panamá Este, Zona Regional de Colón, Zona Regional de Bugaba, Zona Regional de Chiriquí, Zona Regional de Bocas del Toro, Zona Regional de Herrera, Zona Regional de Los Santos, Zona Regional de Veraguas y Zona Regional de Coclé.

Según la Declaración de Principios de la Cumbre Mundial sobre la Sociedad de la Información, en 2003 se comprometió a "Construir una sociedad de la información centrada en las personas y orientada al desarrollo, en la que todo el mundo pueda crear, acceder, utilizar y compartir información y conocimiento".

Otro instrumento lo ofrece la Organización de las Naciones Unidas para la Educación, la Ciencia y la Cultura (UNESCO) son norma de promoción y uso del plurilingüismo y del acceso universal al ciberespacio.

La UNESCO (2005) en la Convención sobre Promoción de la Diversidad Cultural establece que el acceso equitativo a una amplia y diversificada variedad de expresiones culturales, procedentes de todas partes del mundo, y el acceso de las diferentes culturas a los medios de expresión y difusión, son elementos importantes para el mantenimiento de la diversidad cultural y propiciar el entendimiento mutuo. De igual forma la sociedad del aprendizaje y el derecho a la educación.

Como todos sabemos la digitalización como tal toca a todas las formas patrimoniales, artísticas o industriales culturales aunque afecte más profundamente a actividades como bibliotecas, archivos, audiovisual, edición, multimedia. El digital, es un nuevo medio que ofrece grandes ventajas para preservar y, especialmente, difundir los bienes culturales con carácter 
universal. Asimismo es un nuevo ámbito de creación y expresión artística, diferenciado de los clásicos.

Podemos decir también que es una nueva barrera de acceso al uso y disfrute de los bienes culturales en formato digital, si no se ponen en marcha políticas públicas de creación de potentes y accesibles infraestructuras de comunicación y de formación y acceso las tecnologías y se hace un esfuerzo por la calidad y el sentido cultural.

Con todo, la llave de la era digital no es la red o el soporte sino los contenidos, y éstos dependen de la trama productiva cultural previa de un país en los ámbitos creativos, productivos y del uso social, así como de su disposición a asumir los nuevos retos, lo que es especialmente relevante para las culturas minorizadas.

La Cultura Digital es la única temática transversal incluida en los grupos de trabajo por la necesidad de acumular y contrastar experiencias dado su carácter novedoso y su impacto en todos los subsistemas de la cultura. (KULTURAREN; 2013, p. 3-4).

\section{Ciberespacio}

Puede decirse que el ciberespacio es una realidad virtual. No se trata de un ámbito físico, que puede ser tocado, sino que es una construcción digital desarrollada con computadoras.

El escritor norteamericano William Gibson es señalado como quien acuñó la noción de ciberespacio. La utilizó por primera vez en un relato de 1981 y luego ayudó a popularizarla a través de "Neuromante", una novela que publicó en 1984 y que obtuvo el Premio Philip K. Dick, el Premio Hugo y el Premio Nébula.

En la actualidad, el concepto de ciberespacio suele asociarse a Internet. Todo aquello que se desarrolla en Internet, a través de sitios web, correos electrónicos, redes sociales, etc., no tiene lugar en un país específico, más allá de la ubicación concreta de los servidores y de los usuarios. El ciberespacio, de todos modos, es más amplio que Internet.

\section{Cultura digital}

Entendemos por cultura digital como el conjunto de procesos socio-culturales derivados de un contexto emergente en el que las tecnologías digitales son centrales. La cultura digital da pie a "un conjunto de valores, prácticas y expectativas acerca de la manera en que las personas se comportan e interactúan en la sociedad red" (Deuze, 2006). 
Se define por la materialidad de los dispositivos y artefactos que se encuentran en simbiosis con la construcción de sentido, las representaciones sociales, los imaginarios, la identidad. Los sujetos se apropian de las tecnologías digitales y detonan procesos simbólicos y materiales que reconfiguran los sistemas de producción, circulación y consumo de información (Castells, 2010). Las instituciones y las prácticas sociales se transforman, lo cual a su vez determina el curso y la naturaleza del desarrollo tecnológico, que posee la marca del contexto en el que se origina.

\section{Ciberculturas: Nativos e Inmigrantes Digitales.}

El origen del término "Nativos Digitales", proviene de la reflexión del ecléctico Marc Prensky que en su determinante artículo "Digital Natives, Digital Immigrants" de forma concisa define a los nativos digitales como la primera generación que ha crecido con las tecnologías digitales y que son "nativos" del lenguaje de los ordenadores, videojuegos e Internet.

Los nativos digitales son los niños y jóvenes nacidos a partir de 1990, que son expertos con las computadoras, tienen destrezas y formas para comunicarse con los otros que los mayores no pueden entender. Son consumidores y productores de casi todo lo que existe en la red y han provocado una nueva brecha, esta vez alfabeto-generacional.

En cuanto a los inmigrantes digitales son las personas mayores entre 35 y 55 años, nacidos antes de los nativos digitales, es decir en la era pre-digital, por lo que están atados a una lengua materna diferente a la que utilizan los nativos digitales. Han nacido en la era predigital antes de los años 90, suelen escribir y comunicarse de manera correcta y conforme a las reglas ortográficas. Llevan además un seguimiento a las instrucciones antes de llevar a cabo su tarea. Los migrantes digitales son los que han tenido que aprender a usar los medios digitales proviniendo de un mundo analógico de información.

Esta época a la vez es muy interesante ya que se podría decir que aprendieron en una manera muy metódica, estricta no innovadora pero si muy objetiva, a mi punto de vista se puede rescatar de las dos generaciones cosas muy importantes por ejemplo de la inmigrante se debe rescatar el interés por leer, no quedarse con la duda, y de la era nativa el evitar el acudir al plagio hacer la operación de copiar y pegar y no razonar. Sin duda alguna creo que la de los nativos se podría decir hizo que la mayoría de las actividades fueran mucho más fáciles pero menos razonadas. (PRENSKY, 2001)

\section{Signos de la Cultura Digital.}

\section{La Información:}

Exuberancia en una sociedad de carencias. Se considera la información una paradoja que forma parte del ciberespacio. 
Varios autores como (Levy, 1997; Castells 2001) hablan del exceso de información en el ciberespacio, ya que en las décadas recientes se ha generado mayor información que en todos los tiempos de la humanidad.

En otros aspectos Lyoard (1987) considera que la postmodernidad es la era de la información y la caracteriza como símbolo y medio de poder, como un elemento altamente peligroso en una era de desaliento total. Lo es si el contenido y el exceso de la información no se saben manejar.

Algunos aspectos importantes que podemos mencionar sobre la información es que es:

- Tiene alto grado de divulgación

- Disponibilidad

- Exuberancia

- Acceso inmediato

- Ilimitada

- Es un signo de la cibercultura

\section{La Interactividad:}

La interactividad involucra diferentes variables de las cuales las más importantes que se consideran son:

- Fragmentación

- Ponderación en la hipervinculación y

- Extravíos.

Lo digital es interactivo. En la cibercultura existe una lógica que modifica el mundo físico y la interactividad es el factor mediador de ese hecho.

Si el ciberespacio es el depósito de la información digital, con las características que hemos descrito, entonces la cultura digital representa a la arquitectura de la información, un campo de estudio relativamente nuevo en el que encuentran las ciencias de la información y el diseño.

\section{La Hipertextualidad e Hipermeabilidad:}

En el centro del "laberinto de información", como Vattimo (2000) describe la posmodernidad, coloca el desarrollo del escenario multimedia como su eje central. La metáfora de Vattimo evoca un espacio artificial en el que intencionadamente se han combinado encrucijadas. Hasta ahí, la metáfora es epistemológicamente compatible con la noción de las estructuras del hipertexto. 
Ha sido precisamente ese factor: el 'no confundir' a quien entra en estructuras hipertextuales, el que ha guiado las investigaciones sobre usabilidad y ha sido atendido básicamente desde dos áreas:

- El diseño de interfaces y

- la interactividad.

El hipertexto, literalmente, se desplegó y derivó en hipermedia. Como extensión de lo hipertextual, el hipermedia se basa en una concepción tecnológica similar y a diferencia del primero, a éste se integran lenguajes audiovisuales. Es decir, en un hipermedia no sólo hay información textual, sino también visual, sonora, animación y otros lenguajes. Es por ello que se suele relacionar directamente con el concepto multimedia, múltiples medios, múltiples lenguajes. El hipermedia es la articulación, en un espacio multidimensional, de todas las combinaciones posibles de los lenguajes audiovisuales, en una estructura hipertextual y con una variedad casi ilimitada de interconexiones. (Regil, 2000 y 2001).

\section{La Inmediatez:}

La información que se obtiene en la cibercultura se mantiene disponible casi de inmediato, mucha de esta información forma parte de la proliferación y exceso de información del ciberespacio. Es por ello que al buscar cualquier información la misma se mantienen disponible de inmediato.

La inmediatez refiere a proximidad espacial o temporal. Como ya hemos dicho, dos referentes que el ciberespacio modificó y que, en la cultura digital se redimensionan y se resignifican.

\section{La Exclusión:}

Se puede decir que la disponibilidad de las tecnologías digitales y, más aún, su uso especializado, son un factor excluyente en sociedades con economías débiles y bajo nivel educativo; es decir, en donde la exclusión no sólo es digital acusa que la sociedad informacional configuró dos mundos distintos: los que participan y los que quieren participar. Pero no es que la sociedad informacional lo haya dispuesto así, la diferencia se sumó a las desigualdades y exclusiones ya existentes; a estas se añade ahora la digital. En el apartheid digital hay desposeídos digitales. (Castells, 2003).

\section{La Hiperconectividad:}

En la era de las conexiones es la paradoja dentro de este signo. En tiempos de hiperconectividad hay una muchedumbre solitaria (Riesman, Glazer \& Denney, 1964) y 
estamos "conectados pero solos" o "solos en compañía". Pero en la búsqueda de establecer relaciones "sobre todo alimentamos relaciones que podamos controlar, las digitales". (Turkle, 2011, p.14).

La hiperconectividad está estrechamente vinculada a la inmediatez. Ambos signos de la cultura digital se sostienen en la premisa del "aquí y ahora". De hecho, es el signo en donde se sintetizan los demás. Es vehículo de inmediatez para interactuar con información interactiva, hipertextual/hipermedia; y, como indicador del progreso oficial, no tenerla es una de las nuevas formas de exclusión

\section{Las Social Networking:}

El Social Networking son sitios de internet que permiten a las personas conectarse con sus amigos e incluso realizar nuevas amistades, de manera virtual y compartir contenidos, interactuar, crear comunidades sobre intereses similares: trabajo, lecturas, juegos, amistad, relaciones amorosas, relaciones comerciales, etc.

El origen de las redes sociales se remonta al menos a 1995, cuando el estadounidense Randy Conrads creó el sitio Web classmates.com. Con esta red social pretendía que la gente pudiera recuperar o mantener el contacto con antiguos compañeros del colegio, instituto, universidad.

En 2002 comenzaron a aparecer los primeros sitios Web que promocionaban redes de círculos de amigos en línea o relaciones en las comunidades virtuales. La popularidad de estos sitios creció rápidamente y se fueron perfeccionando hasta conformar el espacio de las redes sociales en internet.

Las redes sociales continúan creciendo y ganando adeptos en forma acelerada.

En estas comunidades, un número inicial de participantes envía mensajes a miembros de su propia red social en general su base de contactos de correo electrónico- invitándolos a unirse al sitio. Los nuevos participantes repiten el proceso, y así crecen el número total de miembros y los enlaces de la red.

\section{La Virtualización:}

\section{Web 2.0}

El término Web 2.0 o Web Social comprende aquellos sitios web que facilitan el compartir información, la interoperabilidad, el diseño centrado en el usuario y la colaboración en la World Wide Web.

Un sitio Web 2.0 permite a los usuarios interactuar y colaborar entre sí, como creadores de contenido, en una comunidad virtual. Ejemplos de la Web 2.0 son las comunidades web, los 
servicios web, las aplicaciones Web, los servicios de red social, los servicios de alojamiento de videos, las wikis, blogs, mashups y folcsonomías.

La evolución de las aplicaciones, que pasan de ser estáticas a dinámicas, implica la colaboración del usuario. El término Web 2.0 está asociado estrechamente con Tim O'Reilly, debido a la conferencia sobre la Web 2.0 de O'Reilly Media en 2004.

La Web 2.0 no es más que la evolución de la Web en la que los usuarios dejan de ser usuarios pasivos para convertirse en usuarios activos, que participan y contribuyen en el contenido de la red siendo capaces de crear, dar soporte y formar parte de una sociedad y/o comunidades tanto a nivel local como global; que se informan, comunican y generan conocimiento y contenido.

La Web 2.0 es un concepto que se creó en 2003 y se refiere al fenómeno social surgido a partir del desarrollo de diversas aplicaciones en Internet. El término establece una distinción entre la primera época de la Web (donde el usuario era básicamente un sujeto pasivo que recibía la información o la publicaba, sin que existieran demasiadas posibilidades para que se generara la interacción) y la revolución que supuso el auge de los blogs, las redes sociales y otras herramientas relacionadas.

Se caracteriza principalmente por la participación del usuario como contribuidor activo y no solo como espectador de los contenidos de la Web (usuario pasivo). Esto queda reflejado en aspectos como:

- El auge de los blogs.

- El auge de las redes sociales.

- Las webs creadas por los usuarios, usando plataformas de auto-edición.

- El contenido agregado por los usuarios como valor clave de la Web.

- El etiquetado colectivo (folcsonomía, marcadores sociales, etc).

- La importancia del long tail.

- El beta perpetuo: la Web 2.0 se inventa permanentemente.

- Aplicaciones web dinámicas.

- La World Wide Web como plataforma.

\section{Web 3.0}

La idea de web 3.0, en este contexto, está relacionada a lo que se conoce como web semántica. Los usuarios y los equipos, en este marco, pueden interactuar con la red mediante un lenguaje natural, interpretado por el software. De esta manera, acceder a la información resulta más sencillo. Dicho de otro modo, todos los datos alojados en la web 3.0 deberían ser “entendidos" por las máquinas, que podrían procesarlos con rapidez.

La web 3.0, en definitiva, está relacionada con la inteligencia artificial. Los sitios web incluso tendrían la capacidad de conectarse entre sí de acuerdo a los intereses del usuario. 
En primer lugar, la razón de que no exista una definición formal o bien una única definición de web 3.0 se debe a que muchas personas están trabajando en su propia visión del futuro de Internet, por lo cual cada una espera diferentes cambios y mejoras en la experiencia de navegación por la red.

\section{CONCLUSIONES}

En el presente estudio podemos concluir los siguientes puntos:

- Reforzar la protección de la propiedad intelectual para impulsar la economía del conocimiento generar empleos y consolidar la tecnología utilizada en todas las zonas regionales a nivel nacional.

- Consolidar un verdadero sistema de protección de los derechos en el entorno digital.

- Incentivar la conversión de los modelos de negocio y promover decididamente la oferta legar de los contenidos de la página web.

- Fomentar la digitalización del patrimonio del Benemérito Cuerpo de Bomberos de la República de Panamá, la accesibilidad online, y la preservación de las obras culturales.

- Impulsar normativas que determinen proporcionalidad y equilibrio entre la protección de datos y la protección de los derechos de propiedad individual.

\section{BIBLIOGRAFÍA}

- ÁlvarEZ MONZONCILlO, José María y ZALLO, Ramón.(2002). “Las políticas culturales y de comunicación para el desarrollo de los mercados digitales: un debate necesario", ZER, núm. 13 (noviembre 2002).

- BECERRA, Martín. (2003). Sociedad de la información: proyecto, convergencia, divergencia. Buenos Aires: Grupo Editorial Norma.

- BUSTAMANTE, Enrique. (2002). Comunicación y cultura en la era digital. Industrias, mercados y diversidad en España. Barcelona: Gedisa.

- BUSTAMANTE, Enrique. (2003). Hacia un nuevo sistema mundial de comunicación. Las industrias culturales en la era digital. Barcelona: Gedisa.

- CEPAL. 2003. Informe de la Cumbre Mundial sobre la Sociedad de la Información.

- CHARON, Jean-Marie.(2001). "Entretien avec Jean-Marie Charon: Les incertidumbres du cyberjournalisme”, Sciences humaines, hors-série nº 32, mars 2001, Paris. 
- Guipúzcoa: HIRU Argitaletxe. ZALLO, Ramón.(1988). Economía de la comunicación y la cultura. Madrid: Akal.

- MIÈGE, Bernard; PAJON, Patrick y SALAUN, Jean-Michel.(1986). L'industrialisation de l'audiovisuel. Paris: Editions Aubier. MIÈGE, Bernard (2000): Les industries du contenu face à l'ordre informationnel. Grenoble: Presses Universitaires de Grenoble.

- PlAN Digital 2015. Dirección Nacional de Tecnología, Benemérito Cuerpo de Bomberos de la República de Panamá.

- RAMONET, Ignacio. (2000). La tecnología: revolución o reforma. El caso de la información, (conferencia pronunciada en Pontevedra el 27 de abril de 2000 durante la Semana Galega de Filosofía organizada por el Aula Castelao de Filosofía). 